OPEN ACCESS

Edited by:

Ruth Grene,

Virginia Tech, USA

Reviewed by:

Charu Lata

National Botanical Research Institute

(CSIR), India

Yang Zhao,

Shanghai Institutes for Biological

Sciences (CAS), China

${ }^{*}$ Correspondence:

Shumin Wang

wangshumin@caas.cn

Specialty section: This article was submitted to

Plant Abiotic Stress,

a section of the journal

Frontiers in Plant Science

Received: 20 January 2017

Accepted: 06 March 2017

Published: 23 March 2017

Citation:

Wu J, Chen J, Wang L and Wang S

(2017) Genome-Wide Investigation

of WRKY Transcription Factors Involved in Terminal Drought Stress

Response in Common Bean.

Front. Plant Sci. 8:380

doi: 10.3389/fpls.2017.00380

\section{Genome-Wide Investigation of WRKY Transcription Factors Involved in Terminal Drought Stress Response in Common Bean}

\author{
Jing $W^{1}{ }^{1}$, Jibao $C^{2}{ }^{2}$, Lanfen Wang ${ }^{1}$ and Shumin Wang ${ }^{1 *}$ \\ ${ }^{1}$ Key Laboratory of Crop Gene Resources and Germplasm Enhancement, Ministry of Agriculture/The National Key Facility \\ for Crop Gene Resources and Genetic Improvement/Institute of Crop Science, Chinese Academy of Agricultural Sciences, \\ Beijing, China, ${ }^{2}$ College of Agricultural Engineering, Nanyang Normal University, Nanyang, China
}

WRKY transcription factor plays a key role in drought stress. However, the characteristics of the WRKY gene family in the common bean (Phaseolus vulgaris L.) are unknown. In this study, we identified 88 complete WRKY proteins from the draft genome sequence of the "G19833" common bean. The predicted genes were non-randomly distributed in all chromosomes. Basic information, amino acid motifs, phylogenetic tree and the expression patterns of PvWRKY genes were analyzed, and the proteins were classified into groups 1, 2, and 3. Group 2 was further divided into five subgroups: 2a, 2b, 2c, 2d, and 2e. Finally, we detected 19 WRKY genes that were responsive to drought stress using qRT-PCR; 11 were down-regulated, and 8 were up-regulated under drought stress. This study comprehensively examines WRKY proteins in the common bean, a model food legume, and it provides a foundation for the functional characterization of the WRKY family and opportunities for understanding the mechanisms of drought stress tolerance in this plant.

Keywords: common bean, WRKY transcription factor, drought, qRT-PCR, genome sequence

\section{INTRODUCTION}

The average grain yield of common bean in China is approximately $1,200 \mathrm{~kg} \mathrm{ha}^{-1}$, which is much lower than its potential yields (FAO, 2014). This low productivity is due to biotic and abiotic stress in the farmer's field, such as diseases, insects, and especially drought (Wu et al., 2016). The main common bean planting areas in China, i.e., Heilongjiang, Shanxi, and Guizhou Provinces, have suffered from drought in recent years (Piao et al., 2010). Therefore, there is an urgent need to discover new genes to enhance drought tolerance through molecular breeding.

Transcription factors (TFs) are an important class of genes and include NAM, ATAF1/2, and CUC2 (NAC), basic/helix-loop-helix (bHLH), myeloblastosis (MYB), dehydration responsive element binding protein (DREB), APETALA2/ethyleneresponsive element binding factor (AP2/ERF), and WRKY, among others (Lata et al., 2014; Mao et al., 2015; Dossa et al., 2016; He G.H. et al., 2016; Jin et al., 2016; Zhang et al., 2016). TFs are involved in responses to many types of abiotic and biotic stresses, including drought. The TFs involved in the drought response include ZMNAC111 (maize), TaNAC67 (wheat), TaNAC2 (wheat), OsMYB48-1 (rice), OsDREB2A (rice), 
TaMYBsm1 (wheat), OsAP21 (rice), OsGRAS23 (rice), and others (Cui et al., 2011; Mao et al., 2012, 2014, 2015; Jin et al., 2013; Xiong et al., 2014; Xu et al., 2015; Li M.J. et al., 2016). In the first comprehensive review of the WRKY protein, SPF1 was reported to have been cloned from sweet potato 22 years ago. Since then, many WRKY genes have been found in multiple species, including Arabidopsis, rice, wheat, and soybean (Ishiguro and Nakamura, 1994; Ren et al., 2010; Wang F. et al., 2015; Wang et al., 2016; He Y. et al., 2016; Li W. et al., 2016; Yang et al., 2016). PlantTFDB (V4.0) contains 14,549 WRKY genes from 166 species. The species with the most WRKY genes are Glycine max (296), Brassica napus (285), Panicum virgatum (275), Zoysia matrella (269), and Gossypium hirsutum (238). In contrast, 39 species, including Coffea canephora (49), Genlisea aurea (38), and Carica papaya (49), have fewer than 50 WRKY loci as reported in PlantTFDB.

It is well known that WRKY proteins contain the highly conserved 60 AA WRKY domains (Eulgem et al., 2000; Xie et al., 2005). However, the WRKY amino acid sequences are also replaced by WSKY, WVKY, WKRY, or WKKY in a few WRKY proteins (Xie et al., 2005; Song et al., 2016b). Except for the WRKY domain, WRKY proteins contain zinc finger-like motifs at the $\mathrm{C}$-termini, and the structure is either $\mathrm{Cx}_{4-5} \mathrm{Cx}_{22-23} \mathrm{HxH}$ or $\mathrm{Cx}_{7} \mathrm{Cx}_{23} \mathrm{HxC}$ (Eulgem et al., 2000). WRKY proteins can be divided into three groups. Group I contains two WRKY domains and two zinc finger motifs $\left(\mathrm{Cx}_{4-5} \mathrm{Cx}_{22-23} \mathrm{HxH}\right)$, group II has one WRKY domain and one zinc finger motif $\left(\mathrm{Cx}_{4-5} \mathrm{Cx}_{22-23} \mathrm{HxH}\right)$ and is divided into five subgroups, and group III has one WRKY domain containing one zinc finger motif $\left(\mathrm{Cx}_{7} \mathrm{Cx}_{23} \mathrm{HxC}\right)$ (Rushton et al., 2010; Chen et al., 2012).

Many reports have highlighted the involvement of WRKY proteins in seed development, seed dormancy, seed germination, senescence, development, and biotic and abiotic stress responses (Hinderhofer and Zentgraf, 2001; Robatzek and Somssich, 2001; Johnson et al., 2002; Sun et al., 2003; Zhang et al., 2004; Luo et al., 2005; Eulgem, 2006; Zhou et al., 2008; Li et al., 2009; Popescu et al., 2009; Qiu and Yu, 2009; Tao et al., 2009). Here, we focus on the functional analysis of WRKY proteins in response to abiotic stresses, such as drought. Multiple studies have shown that WRKY genes respond to drought. For example, in Arabidopsis, AtWRKY25, AtWRKY33, AtWRKY46, AtWRKY57, and AtWRKY63 play key roles in the responses to drought stress (Qiu and $\mathrm{Yu}$, 2009; Wu et al., 2009; Song et al., 2010; Ding et al., 2014). Similarly, in rice, overexpression of OsWRKY11, OsWRKY45, and OsWRK72 results in enhanced drought tolerance (Qiu and Yu, 2009; Wu et al., 2009; Song et al., 2010; Ding et al., 2014). In other crops, HvWRKY38, TaWRKY1, TaWRKY33, TaWRKY44, and TaWRKY93 are also involved in the drought response (Marè et al., 2004; Qin et al., 2015; Wang X. et al., 2015). However, compared the grass family, the action of WRKY proteins in legumes is limited. Plants that overexpress GmWRKY54 show enhanced drought tolerance (Zhou et al., 2008).

WRKY proteins have been studied extensively in a variety of plant species (Ishiguro and Nakamura, 1994; Wu et al., 2005;
Li et al., 2009, 2011; Lata et al., 2014; Song et al., 2014, 2016b; He Y. et al., 2016; Wang et al., 2016; Yang et al., 2016). It is important to identify various $\mathrm{TF}$ gene families from whole genome sequences, and many have been identified this way, such as the auxin/indole-3-acetic acid gene family from wheat, AP2/ERF and MYB from foxtail millet, NAC from the common bean, and WRKY from peanut (Lata et al., 2014; Muthamilarasan et al., 2014; Qiao et al., 2015; Wu et al., 2016; Song et al., 2016b). However, a comprehensive view of WRKY proteins in the common bean is still lacking. Recently, the whole genome sequences of the common bean (Andean and Mesoamerican gene pools) have been released, and they are an important resource for the genome-wide analysis of WRKY proteins (Schmutz et al., 2014; Vlasova et al., 2016).

In this study, we performed a genome-wide identification of WRKY TFs in the common bean and analyzed their gene structure, genome distribution, conserved motifs and expression patterns under drought stress in detail. We identified a series of potential candidate WRKY genes related to drought tolerance for future analyses of gene function in the common bean.

\section{MATERIALS AND METHODS}

\section{Plant Material}

In this study, two common bean cultivars, Long 22-0579 (drought-tolerant genotype) and Naihua (drought-sensitive genotype), were used to analyze gene expression patterns under drought stress (Wu et al., 2014). The seeds were obtained from the National Gene Bank (China, Beijing). The seedlings were planted in $23-\mathrm{cm} \times 18-\mathrm{cm}$ (diameter $\times$ depth) plastic pots in a greenhouse under a $14 / 10$-h photoperiod at $25^{\circ} \mathrm{C}$ (day) and $20^{\circ} \mathrm{C}$ (night). All pots were irrigated to field capacity until 4 weeks after seeding. Two treatments, terminal drought and optimal irrigation, were applied. For the terminal drought treatment, watering was restricted to $25 \%$ of field capacity in the pot media from 5 weeks after seeding. For optimal irrigation, the pots were maintained at field capacity throughout the experiment ( $\mathrm{Wu}$ et al., 2014).

\section{Sequence Retrieval and WRKY Gene Identification}

Common bean whole genome sequences and transcript data were downloaded from the Phytozome genome database (Schmutz et al., 2014). The hidden Markov model (HMM) profile of the WRKY family (PF03106) was extracted from the Pfam database (Finn et al., 2016), and the WRKY HMM profile was used to search the common bean whole genome protein database for the WRKY domain using HMMER 3.0 (Finn et al., 2015). All nonredundant sequences hits with expected values lower than $1 \mathrm{E}-5$ were selected and conserved domain checked using PlantTFDB ${ }^{1}$ and SMART $^{2}$ web server.

\footnotetext{
${ }^{1}$ http://planttfdb.cbi.pku.edu.cn/

${ }^{2}$ http://smart.embl-heidelberg.de/
} 


\section{Phylogenetic Analysis}

Phylogenetic trees were generated using MEGA 4.1 software, and cis-acting regulatory elements (CAREs) were identified using the PlantCARE website ${ }^{3}$ (Lescot et al., 2002). Pairwise non-synonymous substitutions rates $(K s)$ and synonymous substitutions rates $(\mathrm{Ka})$ were calculated using codeml in PAML 4.3a with the F3 $\times 4$ codon frequency model (Yang, 2007). The divergence time was calculated using the formula $T=K s / 2 \lambda$, assuming a common bean and soybean substitution rate $(\lambda)$ of $6.1 \times 10^{-9}$ substitutions/synonymous site/year (Egan and Doyle, 2010).

\section{Analysis of Protein Features}

ExPASy was used to determine the basic WRKY gene information [molecular weight (MW), number of amino acids, open reading frame (ORF), ORF length, and isoelectric point $\left.(\mathrm{pI})^{4}\right]$. Multiple alignment analysis was performed using ClustalW ${ }^{5}$, and subcellular localization was predicted using the softberry website $^{6}$. The structure of the WRKY genes was investigated using the Gene Structure Display Server websites ${ }^{7}$.

\section{Quantitative RT-PCR}

Leaves were obtained from 10 individual plants as they began to wilt under drought stress, immediately frozen in liquid nitrogen and stored at $-80^{\circ} \mathrm{C}$. Four samples were designated as LOI, LTD, NOI, and NTD according to cultivar (Long 22-0579 [L] or Naihua [N]) and treatment (optimal irrigation [OI] or terminal drought [TD]). Total RNA was extracted using TRIzol reagent (Tiangen, Beijing) according to the manufacturer's instructions. The quality and quantity of RNA was evaluated by agarose gel electrophoresis and NanoDrop (Thermo Fisher Scientific, Waltham, MA, USA), respectively. For first-strand cDNA synthesis, $1 \mu \mathrm{g}$ of total RNA after DNA enzyme digestion was synthesized using the SuperScript ${ }^{\circledR}$ II reverse transcriptase kit following the manufacturer's protocols (Invitrogen, USA).

The qRT-PCR reactions were performed with an ABI PRISM 7300 Sequence Detection System (Thermo Fisher Scientific, Waltham, MA, USA) as follows: $95^{\circ} \mathrm{C}$ for $30 \mathrm{~s}$ followed by 40 cycles of $95^{\circ} \mathrm{C}$ for $5 \mathrm{~s}$ and $60^{\circ} \mathrm{C}$ for $31 \mathrm{~s}$ using $\mathrm{SYBR}^{\circledast}$ Premix Ex Taq ${ }^{\mathrm{TM}}$ (TaKaRa, Tokyo, Japan). A melting curve was generated as follows: $95^{\circ} \mathrm{C}$ for $15 \mathrm{~s}, 60^{\circ} \mathrm{C}$ for $60 \mathrm{~s}$, and $95^{\circ} \mathrm{C}$ for $15 \mathrm{~s}$.

All reactions were performed in triplicate, and the relative expression levels were calculated using the $2^{-\Delta \Delta C T}$ method with normalization to the internal control gene, Skip16 (Borges et al., 2012). The specific gene primers are listed in Supplementary Table S1 and were designed with Primer 5.0 software. In this study, differentially expressed genes with higher expression levels in TD samples than in OI samples were denoted by "upregulated," and those with lower expression levels were denoted by "down-regulated."

\footnotetext{
${ }^{3}$ http://bioinformatics.psb.ugent.be/webtools/plantcare/html/

${ }^{4}$ http://www.expasy.ch/tools/pi_tool.html

${ }^{5} \mathrm{http}: / /$ www.genome.jp/tools/clustalw/

${ }^{6} \mathrm{http}: / /$ linux 1. softberry.com/

${ }^{7}$ http://gsds.cbi.pku.edu.cn/
}

\section{RESULTS}

\section{Identification and Distribution of the WRKY Proteins in the Common Bean}

A total of 102 candidate genes corresponding to the Pfam WRKY family were obtained with the HMM (Supplementary Table S2), and 90 non-redundant WRKY genes were identified in the common bean genome, of which 88 full-length protein sequences were used for further analyses (Supplementary Table S3). A total of 88 WRKY proteins were identified from the common bean (G19833) using a bioinformatics approach (Supplementary Table S3). Gene characteristics, including the length of the full-length sequence, length of the CDS, length of the protein sequence, gene MW, pI, subcellular localization and the corresponding positions, were analyzed (Supplementary Table S3). These genes were named PvWRKY1 to PvWRKY88. The length of all fulllength sequences ranged from 685 (PvWRKY72) to 7,722 bp (PvWRKY62), with an average of 2,340 bp. The length of the CDS ranged from 450 (PvWRKY23) to 2,223 bp (PvWRKY34), with an average of $1143 \mathrm{bp}$. The length of the protein sequences ranged from 149 (PvWRKY23) to $740 \mathrm{AA}$ (PvWRKY34), with an average of $380 \mathrm{AA}$. The gene MW ranged from 17.36 (PvWRKY1) to $80.20 \mathrm{kDa}$ (PvWRKY34), with an average of $42.07 \mathrm{kDa}$; and the pI ranged from 4.83 (PvWRKY7) to 9.94 (PvWRKY29) with an average of 7.07 . The predicted subcellular localization results indicated that 65 PvWRKY proteins were located in the nuclear region, whereas the remaining proteins were located in the extracellular region.

Figure 1 shows that all common bean WRKY genes are distributed across all 11 chromosomes (Ch1-Ch11). The distribution of these genes is uneven. Some chromosomes (e.g., Chr 2 and Chr 8, which represent $18.2 \%$ of the WRKY genes) have more genes, whereas others have few (e.g., $\mathrm{Chr} 9$ and $\mathrm{Chr} 1$ ); some chromosomes have only one gene (Chr 4 and $\mathrm{Chr} 11)$; and some of these genes are clustered. To form a cluster, the distance between neighboring WRKY genes had to be less than $200 \mathrm{~kb}$, and separated by no more than eight non-WRKY genes between the WRKY genes (Lozano et al., 2015). We identified 11 small clusters containing 24 WRKY genes, and the average number of WRKY genes in a cluster was 2.2 (Supplementary Table S3). Most of the clusters had two members; only clusters 4 and 8 had three members. Cluster size ranged from 9,066 to 823,695 bp. Cluster 3 was the longest cluster, and cluster 10 was the shortest cluster.

\section{Multiple Sequence Alignment and Structure Analysis}

WRKY proteins contain two very conservative and important motifs, the first of which is the WRKYGQK sequence that always recognizes and binds to the $\mathrm{W}$-box element. In addition to the WRKYGQK sequences, four variants, WRKYGKK, WRKYGEK, WKKYEDK, and WKKYCEDK, were observed in the common bean WRKY proteins (Supplementary Figure S1 and Table S3). The WRKYGQK sequences represented the major variant in PvWRKY proteins at approximately $90.1 \%$. The second motif is a zinc finger structure containing two types of zinc finger motifs: $\mathrm{Cx}_{4-5} \mathrm{Cx}_{22-23} \mathrm{HxH}$ and $\mathrm{Cx}_{7} \mathrm{Cx}_{23} \mathrm{HxC}$, both of which were 


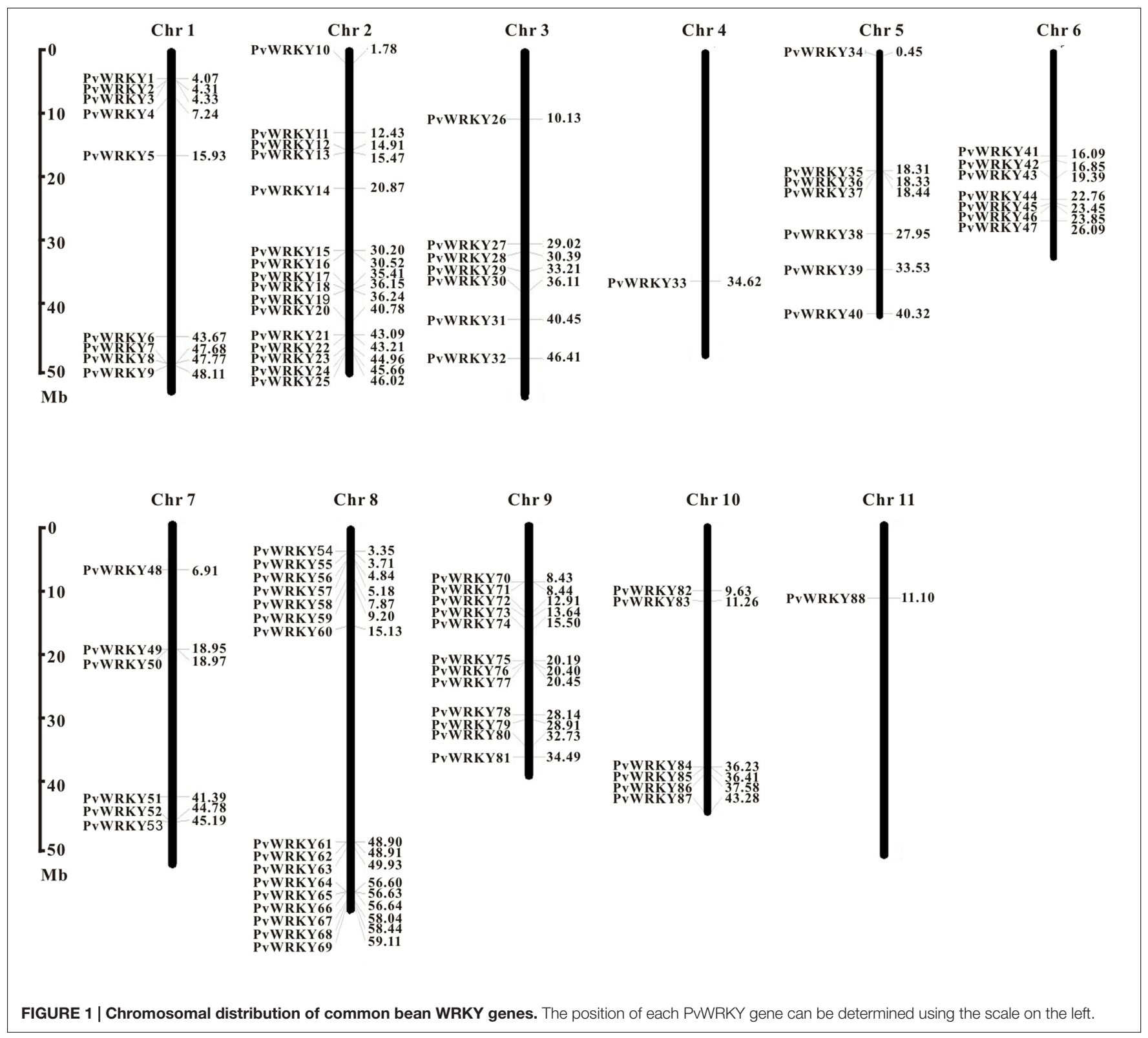

observed in the common bean WRKY proteins. Furthermore, 74 PvWRKY proteins contained $\mathrm{Cx}_{4-5} \mathrm{Cx}_{22-23} \mathrm{HxH}$-type zinc finger motifs, and $14 \mathrm{PvWRKY}$ proteins contained $\mathrm{Cx}_{7} \mathrm{Cx}_{23} \mathrm{HxC}$ type zinc finger motifs (Supplementary Figure S1 and Table S3).

The PvWRKY proteins could all be divided into three groups, 1, 2, and 3, containing 15, 57, and 14 proteins, respectively. Notably, PvWRKY65 and PvWRKY66 were not assigned to any group and existed alone because of their unique WRKY sequences (WKKY/CEDK). To better separate the groups, a phylogenetic tree was generated based on the protein sequences of all the PvWRKY genes and 35 homologous soybean WRKY proteins, and the WRKY genes were divided into three groups: 1 (15 sequences), 2 (57 sequences), and 3 (14 sequences) (Figures 2A, 3). Fifty-five sequences from group 2 were further classified into five subgroups: $2 \mathrm{a}$ (5 sequences), 2b (15 sequences), 2c (19 sequences), 2d (8 sequences), and $2 \mathrm{e}$ (10 sequences). Similar results were also observed in soybean (Song et al., 2016a). Furthermore, each group had a different WRKYGQK sequence and zinc finger motifs; group 1 had two WRKYGQK sequences and two zinc finger motifs. The WRKYGQK sequence of all PvWRKY proteins except PvWRKY 83 from this group was WRKYGQK, and all zinc finger motifs at the $\mathrm{N}$-terminus were $\mathrm{CxX}_{4} \mathrm{Cx}_{22} \mathrm{Hx}_{1} \mathrm{H}$. In contrast, all zinc finger motifs at the C-terminus were $\mathrm{Cx}_{4} \mathrm{Cx}_{23} \mathrm{Hx}_{1} \mathrm{H}$. In group 2, subgroups $2 \mathrm{a}, 2 \mathrm{~b}, 2 \mathrm{~d}$, and $2 \mathrm{e}$ had the same motif, WRKYGQK and $\mathrm{Cx}_{5} \mathrm{Cx}_{23} \mathrm{Hx}_{1} \mathrm{H}$. Notably, the WRKYGQK sequence in the subgroup 2c WRKY proteins showed several variations: WRKYGQK was detected in most of the subgroup 2c WRKY proteins; WRKYGKK was detected in PvWRKY1, PvWRKY21, PvWRKY72, and PvWRKY77; and 


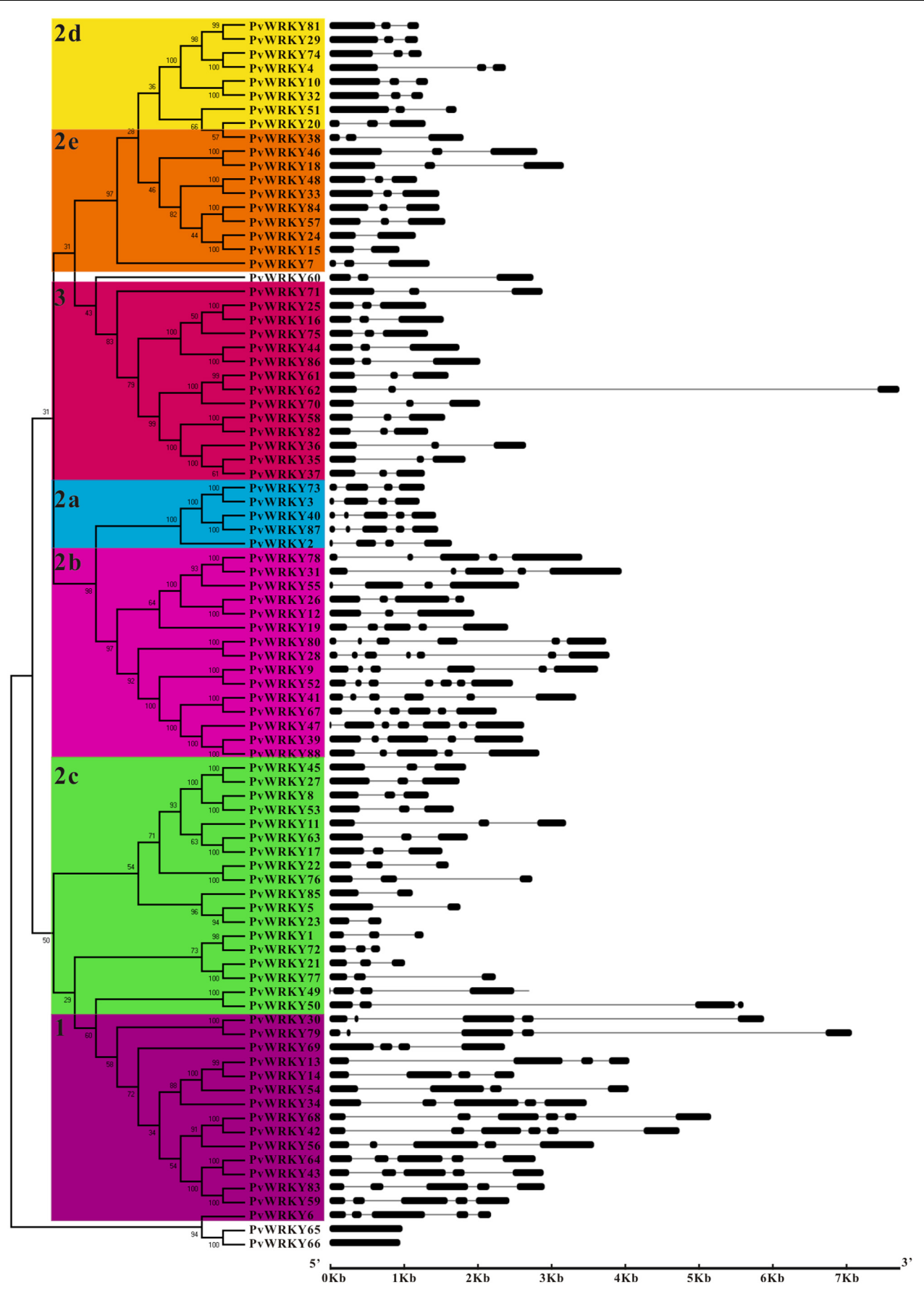

FIGURE 2 | Phylogenetic tree and gene structure of common bean WRKY genes. (A) The phylogenetic tree was constructed with MEGA 4.1 software by the neighbor-joining (NJ) method with 1,000 bootstrap replicates. (B) Exon/intron structure of PVWRKY genes: the introns and exons are represented by gray lines and black boxes, respectively.

WRKYGEK was detected in PvWRKY23. However, the PvWRKY proteins from group 3 had a consistent motif: WRKYGQK and $\mathrm{Cx}_{7} \mathrm{Cx}_{23} \mathrm{Hx}_{1} \mathrm{C}$.

A detailed illustration of the gene structures is shown in Figure 2B and Supplementary Table S3. The number of introns ranged from one to six in all members of the common bean
WRKY gene family except PvWRKY65 and PvWRKY66, and the average number of exons among the full-length WRKY genes in the common bean genome was 3.73. Most PvWRKY genes contained the typical splicing of three exons and two introns (45 of the 88 PvWRKY genes); however, PvWRKY28, PvWRKY47, and PvWRKY52 had the greatest numbers, seven exons and six 


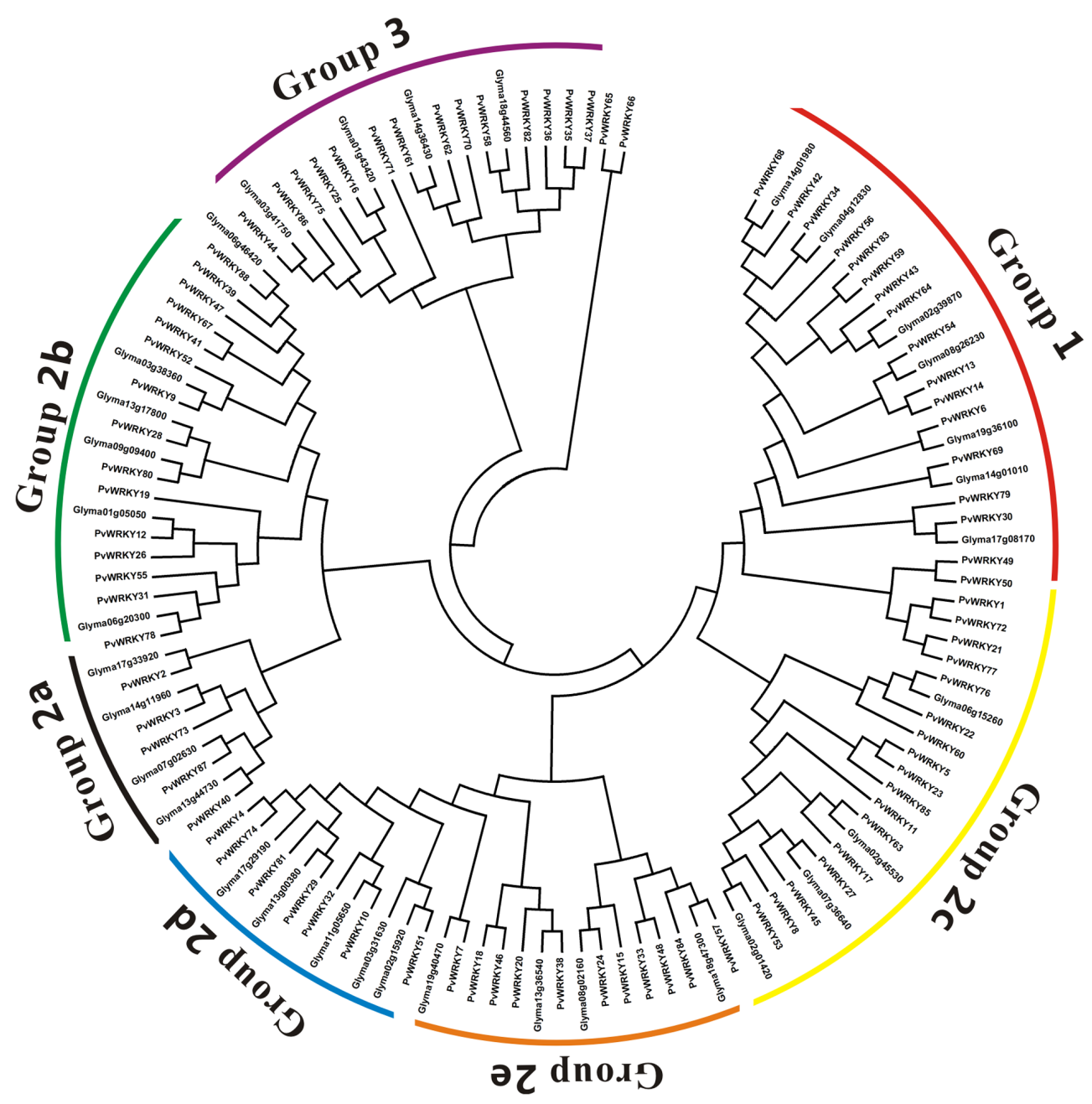

FIGURE 3 | Phylogenetic tree of PvWRKY proteins and homologous soybean WRKY proteins. The phylogenetic tree was constructed using same method as used for Figure 2A.

introns, whereas PvWRKY65 and PvWRKY66 each harbored one exon. Overall, the phylogenetic analysis of the PvWRKY gene family showed that genes within the same group generally had a similar structure (Figure 2B); for example, subgroups $2 \mathrm{~d}$ and 2e contained three exons and two introns.

\section{Cis-Acting Regulatory Element Analysis of the WRKY Promoter}

We analyzed 1500-bp sequences upstream of the translational start site (Supplementary Table S4) and divided these elements into seven groups: essential element, enhancer, light responsive, tissue-specific expression, abiotic stress, hormone, and other types of elements. In putative essential CAREs, TATA, and CAAT boxes were detected upstream of the transcription start site. The light-responsive type had the highest number of members, harboring 35 elements including Gap-box, AEbox, ATCT-motif, and Box-4. Some CAREs involved in the tissue-specific expression of root, shoot, seed, and meristem were found in the promoter regions of WRKY genes, such as AACA_motif, which is related to endosperm-specific negative expression, and CCGTCC-box, which is involved in meristem-specific activation. The most noteworthy result is that the CAREs associated with abiotic or biotic stress and the related hormones were found in the promoter region; ABRE is involved in abscisic acid responsiveness; P-box, TATC-box, and GARE-motif are gibberellin-responsive elements; TCA-element and SARE are involved in salicylic acid responsiveness; CGTCA-motif and TGACG-motif are involved in MeJA responsiveness; TGA-element and AuxRRcore are involved in auxin responsiveness; $\mathrm{MBS}$ is involved in drought inducibility; and DRE and LTR are involved in the responses to dehydration, low temperatures, and salt stress. Furthermore, we observed that more than one abiotic or biotic stress and hormone element occur in one promoter region; for example, PvWRKY67 contained five abiotic or biotic stress elements (LTR, HSE, MBS, WUN-motif, and TC-rich repeats) and four hormone elements (ELI-box3, P-box, CGTCA-motif, TGA-element, and TGACG element), and PvWRKY32 included 


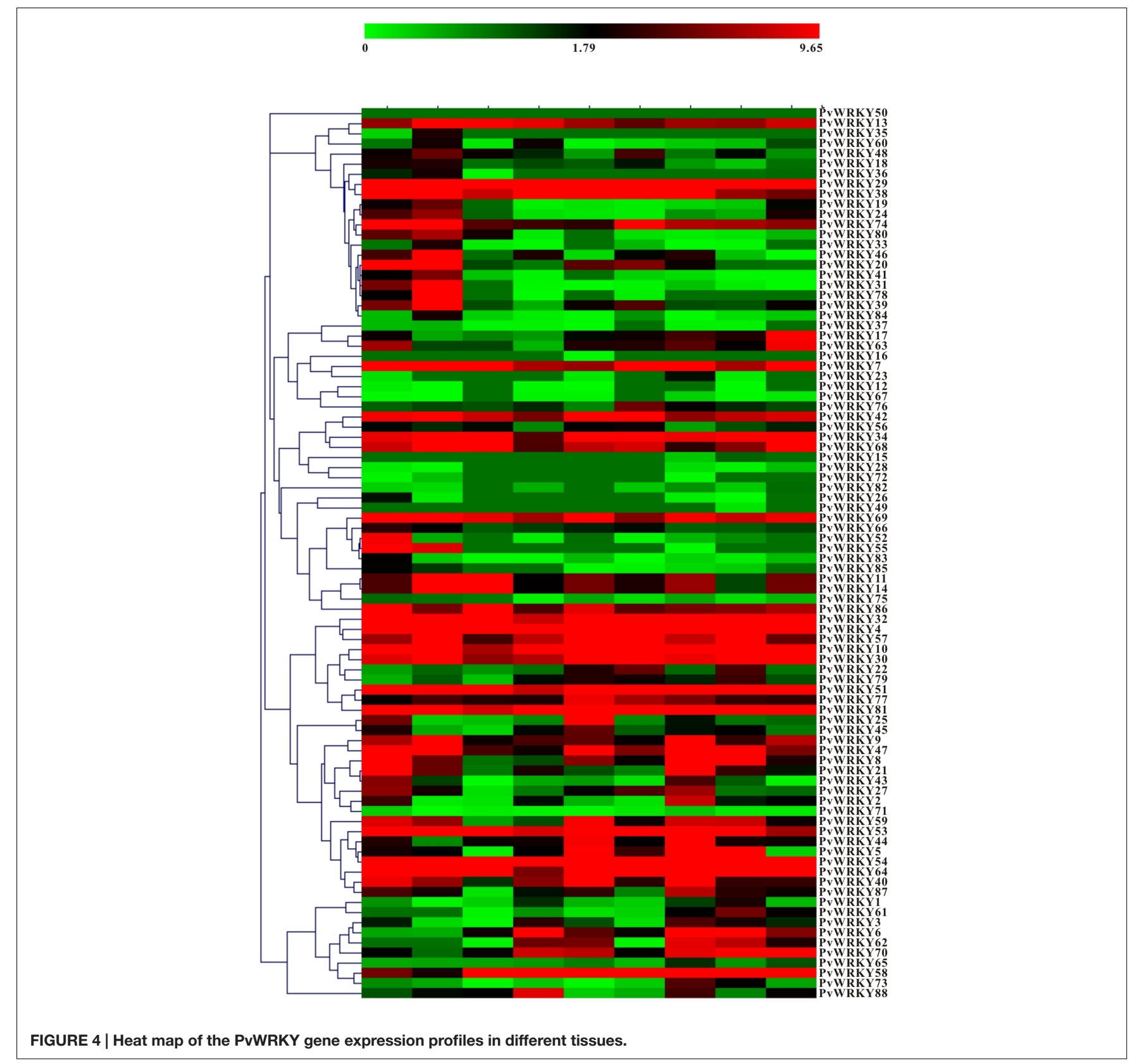

four abiotic or biotic stress elements (HSE, MBS, WUNmotif, and DRE) and six hormone elements (TCA-element, ABRE, P-box, GARE-motif, CGTCA-motif, and TGACG element).

\section{Evolutionary Patterns and Divergence of the WRKY Gene Family between the Common Bean and Soybean}

The common bean and soybean belong to the legume family and are closely related. In this paper, we determined the $\mathrm{Ka} / \mathrm{Ks}$ substitution ratio in the coding sequences of orthologs between the WRKY families of the common bean and soybean (Supplementary Table S5). All pairwise $\mathrm{Ka} / \mathrm{Ks}$ ratios were below 1 and ranged from 0.0937 to 0.9787 , suggesting that the PvWRKY genes are under purifying selection. To confirm the divergence time between the common bean and soybean, divergence times were calculated with relative $K s$ values. Most of the $K s$ values concentrated at $0.2-0.3$, suggesting a largescale event approximately 16.1-24.2 million years ago (MYA). A previous study estimated that common bean and soybean diverged 19.2 MYA (Lavin et al., 2005).

\section{Transcriptome Atlas Analysis of the WRKY Gene Family}

The availability of transcriptome data facilitates study of the basic biology of the common bean. Expression data were obtained 


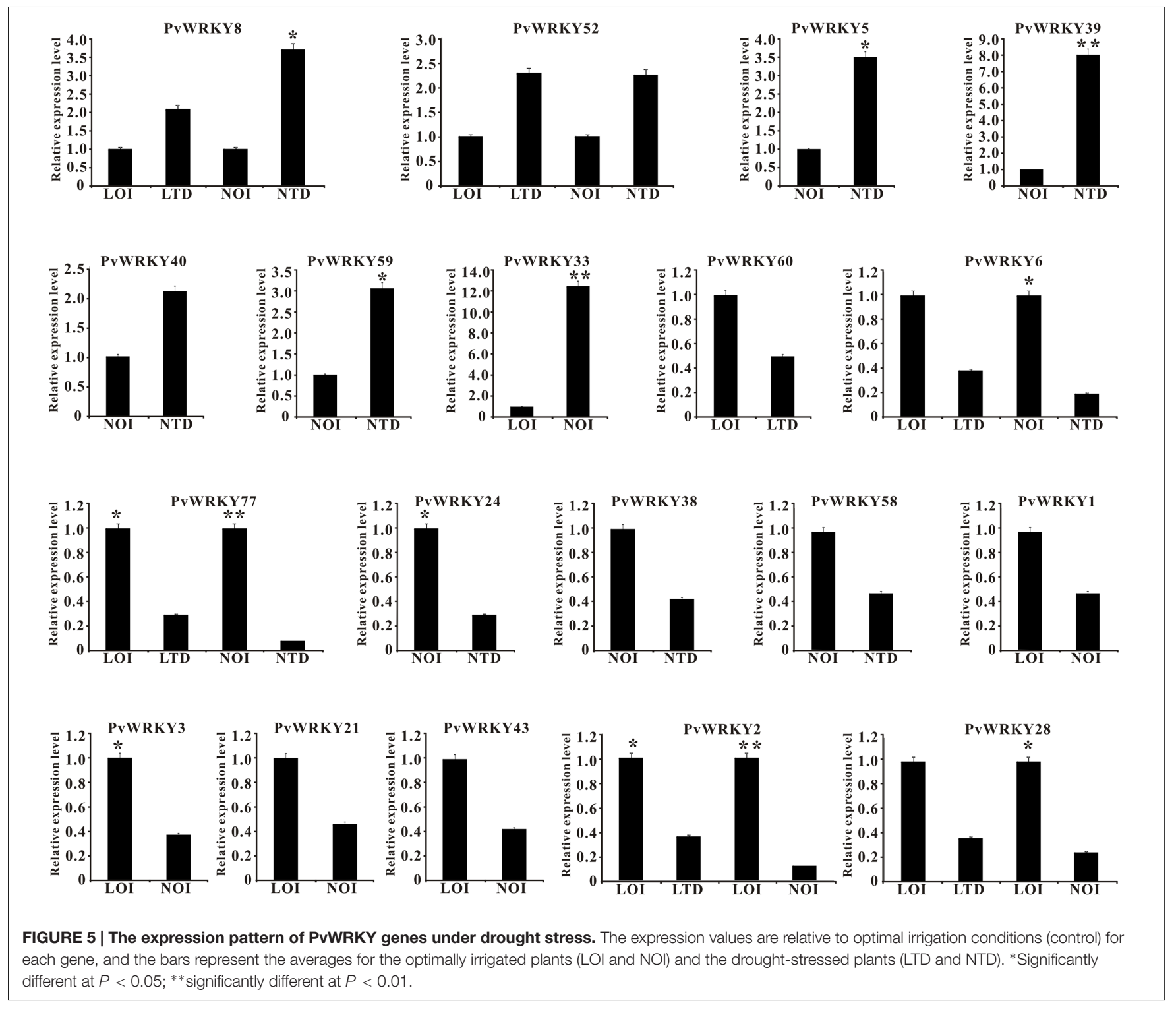

for 87 PvWRKY TFs in nine tissues including young trifoliates, leaves, flower buds, flowers, green mature pods, young pods, roots, stems, and nodules (Figure 4 and Supplementary Table S6). However, PvWRKY50 lacked expression data. Approximately 93.18\% of the PvWRKYs were expressed in nodules, followed by flower and young pods $(92.05 \%)$, roots $(90.91 \%)$, and flower buds (86.36\%); few WRKY genes were detected in leaves (78.41\%). In the common bean, $69.3 \%$ (61 of 88 ) of the PvWRKY proteins were constitutively expressed in every tested tissue. Furthermore, PvWRKY16 and PvWRKY49 were specifically expressed in green mature pods and young pods, respectively. PvWRKY15 was co-expressed in flowers and young pods, whereas PvWRKY35 was co-expressed in nodules and roots. Moreover, PvWRKY4, PvWRKY10, PvWRKY29, PvWRKY32, PvWRKY34, PvWRKY51, PvWRKY54, PvWRKY64, and PvWRKY81 were highly expressed in the vast majority of common bean tissues.

\section{Expression Profiles of the WRKY Gene Family under Drought Stresses}

To determine the expression profiles of the PvWRKY gene family under drought stress, 88 PvWRKY genes were analyzed by qRT-PCR. The expression of the PvWRKY genes was significantly altered (fold change $\geq 2$ ) under drought stress. We obtained 19 PvWRKY genes showing different expression levels under drought stress, including 7 up-regulated and 12 down-regulated genes (Figure 5 and Table 1). Seven WRKY genes showed different expression levels between droughttolerant and drought-sensitive genotypes. Among these genes, only PvWRKY33 showed a lower expression level in the droughttolerant genotype than in the drought-sensitive genotype. For the drought-tolerant genotype, PvWRKY8 and PvWRKY52 were up-regulated under drought stress, and five WRKY genes were down-regulated. For the drought-sensitive genotype, six 
TABLE 1 | The differential expression levels of PvWRKY genes.

\begin{tabular}{|c|c|c|c|c|c|}
\hline Expression pattern & & Genes & Fold change (LOI to LTD) & Fold change (NOI to NTD) & Fold change (LOI to NOI) \\
\hline & LOI/NOI and NOI/NTD & PvWRKY52 & 2.28 & 2.25 & \\
\hline & \multirow{3}{*}{ NOI/NTD } & PvWRKY39 & & 8.14 & \\
\hline & & PvWRKY40 & & 2.09 & \\
\hline & & PvWRKY59 & & 3.08 & \\
\hline \multirow[t]{9}{*}{ Down-regulated } & LOI/LTD & PvWRKY60 & -2.02 & & \\
\hline & \multirow[t]{2}{*}{ LOI/NOI and NOI/NTD } & PvWRKY6 & -2.62 & -5.39 & \\
\hline & & PvWRKY77 & -3.41 & -12.10 & \\
\hline & \multirow[t]{3}{*}{ NOI/NTD } & PvWRKY24 & & -3.43 & \\
\hline & & PvWRKY38 & & -2.40 & \\
\hline & & PvWRKY58 & & -2.09 & \\
\hline & LOI/NOI & PvWRKY43 & & & -2.33 \\
\hline & \multirow[t]{2}{*}{ LOI/LTD and LOI/NOI } & PvWRKY2 & -2.71 & & -8.08 \\
\hline & & PvWRKY28 & -2.76 & & -4.07 \\
\hline
\end{tabular}

WRKY genes were up-regulated under drought stress, and five were down-regulated. Four genes showed the same expression pattern in the drought-sensitive and drought-tolerant genotypes under drought stress: PvWRKY8 and PvWRKY52 were upregulated, and PvWRKY6 and PvWRKY77 were down-regulated. PvWRKY2 and PvWRKY28 were down-regulated between LOI/LTD and LOI/NOI.

\section{DISCUSSION}

WRKY proteins have been detected in various organisms, such as spike mosses, single-celled green algae, slime molds and protozoa (Rushton et al., 2010). In monocots and dicots, such as rice, soybean, wheat, and cotton, an especially large number of WRKY proteins have been confirmed to have various functions in recent years (Qiu and Yu, 2009; Luo et al., 2013; Qin et al., 2015; He G.H. et al., 2016; Liu et al., 2016). However, our study is the first to identify and characterize WRKY proteins from whole genome sequences of the common bean.

An increasing number of whole plant genomes have been sequenced, and an increasing number of WRKY genes have been identified in plant species (Yin et al., 2013; He Y. et al., 2016; Song et al., 2016a,b; Wei et al., 2016; Yue et al., 2016). Completion of the common bean genome makes it possible to analyze WRKY TFs at the whole genome level (Schmutz et al., 2014), and in this study, we systematically identified 88 WRKY members in common bean accession G19833. The most obvious variation occurred in CDS length, protein length, PI value, MW, and other basic information, which is consistent with the WRKY family in other plants, such as peanut (Song et al., 2016b). The distributions of the PvWRKY genes across the chromosome appeared to be non-random, as they always formed a gene cluster, which is similar to the results for the WRKY family in other plants and previous genome-wide reports on NAC genes in the common bean (Song et al., 2016a,b; Wu et al., 2016; Yue et al., 2016). Furthermore, in previous studies, WRKY gene numbers varied among species; 74 WRKY proteins have been identified in A. thaliana (Ülker and Somssich, 2004), 102 in Oryza sativa ssp. indica (Ross et al., 2007), 97 in Oryza sativa ssp. japonica (Rushton et al., 2010), and 75 in Medicago truncatula (Rushton et al., 2010). These numbers are similar to the number of WRKY genes in the common bean. In contrast, the number of WRKY genes in soybean (197) (Schmutz et al., 2010) is twice that in the common bean. This difference may be related to species differences in the size of the genome: common bean, $587 \mathrm{Mb}$ (Schmutz et al., 2014); Arabidopsis, 119 Mb (The Arabidopsis Genome Initiative, 2000); Oryza sativa ssp. indica, $466 \mathrm{Mb}$ (Yu et al., 2002); Oryza sativa ssp. japonica, 420 Mb (Goff et al., 2002); Medicago truncatula, $500 \mathrm{Mb}$ (Young et al., 2011); and soybean, $1100 \mathrm{Mb}$ (Schmutz et al., 2010). Therefore, the abundance of WRKY genes has expanded, which may be a result of genome duplications. In the common bean, PvWRKY proteins can be divided into three groups, and group 2 can be divided into five subgroups, $2 \mathrm{a}, 2 \mathrm{~b}, 2 \mathrm{c}, 2 \mathrm{~d}$, and $2 \mathrm{e}$, based on the amino acid sequences outside the WRKY domain. We also observed that the number of proteins in subgroup $2 \mathrm{c}$ was the highest among all subgroups. These results are consistent with the results for other species (Yin et al., 2013; He G.H. et al., 2016; Song et al., 2016a,b; Wei et al., 2016; Yue et al., 2016). However, the variants WRKYGKK, WRKYGEK, WKKYEDK, and WKKYCEDK were mainly observed in subgroup $2 c$ of the common bean, suggesting that the WRKY proteins of subgroup $2 c$ may have a variety of biological functions. The expression 
profiles revealed different expression patterns for each PvWRKY gene in different tissues, providing a valuable resource for gene functional research. Most PvWRKY genes were expressed in all nine tissues, and nine PvWRKY genes were highly expressed, which suggests that these PvWRKY genes may be essential for plant growth. However, several PvWRKY genes were expressed only in one specific tissue, suggesting that these genes might have tissue-specific functions. The expression profiles generated in this study provide very rich data resources to further investigate the function of PvWRKY genes.

Among the TF families in higher plants, WRKY TFs have been found to play important roles under biotic and abiotic stress, especially drought (Ding et al., 2014; Qin et al., 2015; He Y. et al., 2016; Liu et al., 2016; Yue et al., 2016). Using qRT-PCR in the present study, we identified 19 common bean WRKY TFs that were responsive to drought stress. Among these, 10 PvWRKYs contain MBS elements involved in drought inducibility, such as PvWRKY1, PvWRKY21, PvWRKY28, PvWRKY24, and PvWRKY52. We also found that six PvWRKYs contain ABRE elements involved in drought stress tolerance (Song L. et al., 2016), including PvWRKY2, PvWRKY3, PvWRKY5, PvWRKY38, PvWRKY43, and PvWRKY58. These results support the qRT-PCR results. Furthermore, these genes can be divided two groups: those that were differentially expressed between drought-tolerant and drought-sensitive genotypes and those that were differentially expressed between the treatment and the control. These genes may be good candidates for enhancing drought stress tolerance because we can putatively predict the function of PvWRKY genes based on their identified homologous genes (Supplementary Figure S2). For example, GmWRKY27, a PvWRKY40 homolog, improves drought tolerance in transgenic soybean (Wang F. et al., 2015), and GmWRKY54, a PvWRKY53 homolog, confers drought tolerance (Zhou et al., 2008). Interestingly, there have been no reports regarding the functions of several PvWRKY homolog genes, such as PvWRKY5, PvWRKY33, and PvWRKY58; therefore, there may be additional WRKY TFs that are involved in drought resistance in the common bean. However, we also found several PvWRKY genes that were not induced by drought stress but have homologs in other species that function under drought stress. It is possible that the expression of these genes differed only slightly between drought-tolerant and drought-sensitive genotypes. However, different PvWRKYs play different roles in regulating the stress response; therefore, further investigation into their expression patterns under different stresses (salt, heat, and low temperature) is necessary. The results reported here provide some candidates for future studies of the drought resistance mechanism.

\section{REFERENCES}

Borges, A., Tsai, S. M., and Caldas, D. G. G. (2012). Validation of reference genes for RT-qPCR normalization in common bean during biotic and abiotic stresses. Plant Cell Rep. 31, 827-838. doi: 10.1007/s00299-011-1204-x

Chen, L. G., Song, Y., Li, S. J., Zhang, L. P., Zou, C. S., and Yu, D. Q. (2012). The role of WRKY transcription factors in plant abiotic stresses. Biochim. Biophys. Acta 1819, 120-128. doi: 10.1016/j.bbagrm.2011.09.002
In this study, the phylogenetic relationships, exon/intron structures, and expression pattern of WRKY family members under drought stress were evaluated in the common bean, and we identified 19 PvWRKY genes that are responsive to drought stress. As only a few WRKY genes have been detected in the common bean to date, our results will facilitate the functional analysis of PvWRKY genes. These results offer a useful resource for understanding the potential physiological role of individual WRKY genes during drought stress.

\section{AUTHOR CONTRIBUTIONS}

JW and SW conceived the experiments and analyzed the data, and JC and LW performed the experiments. JW and SW contributed to the writing of the manuscript, which all the authors reviewed.

\section{FUNDING}

This work was supported by grants from the National Natural Science Foundation of China (grant No. 31471559), the Ministry of Agriculture of China [the earmarked fund for the China Agriculture Research System (CARS-09)], the National Key Technology Research and Development Program of the Ministry of Science and Technology of China (2013BAD01B03-18a) and the Agricultural Science and Technology Innovation Program (ASTIP) of CAAS.

\section{SUPPLEMENTARY MATERIAL}

The Supplementary Material for this article can be found online at: http://journal.frontiersin.org/article/10.3389/fpls.2017.00380/ full\#supplementary-material

\section{FIGURE S1 | Alignment of multiple common bean WRKY genes.}

FIGURE S2 | Phylogenetic tree of PvWRKY and drought-related WRKY proteins from other species. The phylogenetic tree was constructed with MEGA4.1 software using the NJ method and 1,000 bootstrap replicates. The green dots represent drought-related WRKY proteins from other species, and the red squares represent drought-related PVWRKY proteins. The accession numbers of all drought-related WRKY proteins follow: TaWRKY93 (JX679079), TaWRKY33 (KT285207), HvWRKY38 (CAD60651), GmWRKY27 (DQ322695), GhWRKY15 (GU207869), GmWRKY54 (DQ322698), AtWRKY57 (AT1G38010), WRKY25 (JF899343), GhWRKY3 (FJ966887), TaWRKY19 (EU665430), OsWRKY30 (ABC02808), TaWRKY2 (EU665425), TaWRKY1 (KT285206), TcWRKY53 (EF053036), AtWRKY63 (AT1G66600), OsWRKY45 (BK065048), AtWRKY46 (AT2G46400), OsWRKY08 LOC_Os05g50610), OsWRKY11 (LOC_Os01g43650), GmWRKY13 (DQ322694), GmWRKY21 (DQ322691).

Cui, M., Zhang, W., Zhang, Q., Xu, Z., Zhu, Z., Duan, F., et al. (2011). Induced overexpression of the transcription factor OsDREB2A improves drought tolerance in rice. Plant Physiol. Biochem. 49, 1384-1391. doi: 10.1016/j.plaphy.2011. 09.012

Ding, Z. J., Yan, J. Y., Xu, X. Y., Yu, D. Q., Li, G. X., Zhang, S. Q., et al. (2014). Transcription factor WRKY46 regulates osmotic stress responses and stomatal movement independently in Arabidopsis. Plant J. 79, 13-27. doi: 10.1111/tpj. 12538 
Dossa, K., Wei, X., Li, D., Fonceka, D., Zhang, Y., Wang, L., et al. (2016). Insight into the AP2/ERF transcription factor superfamily in sesame and expression profiling of DREB subfamily under drought stress. BMC Plant Biol. 16:171. doi: 10.1186/s12870-016-0859-4

Egan, A. N., and Doyle, J. (2010). A comparison of global, gene-specific, and relaxed clock methods in a comparative genomics framework: dating the polyploid history of soybean (Glycine max). Syst. Biol. 59, 534-547. doi: 10.1093/sysbio/ syq041

Eulgem, T. (2006). Dissecting the WRKY web of plant defense regulators. PLoS Pathog. 2:e126. doi: 10.1371/journal.ppat.0020126

Eulgem, T., Rushton, P. J., Robatzek, S., and Somssich, I. E. (2000). The WRKY superfamily of plant transcription factors. Trends Plant Sci. 5, 199-206. doi: 10.1016/S1360-1385(00)01600-9

FAO (2014). FAO Statistical Yearbook. Rome: FAO. Available at: http://faostat3.fao. org/browse/Q/QC/E

Finn, R. D., Clements, J., Arndt, W., Miller, B. L., Wheeler, T. J., Schreiber, F., et al. (2015). HMMER web server: 2015 update. Nucleic Acids Res. 43, W30-W38. doi: 10.1093/nar/gkv397

Finn, R. D., Coggill, P., Eberhardt, R. Y., Eddy, S. R., Mistry, J., Mitchell, A. L., et al. (2016). The Pfam protein families database: towards a more sustainable future. Nucleic Acids Res. 44, D279-D285. doi: 10.1093/nar/gkv1344

Goff, S. A., Darrell, R., Tien-Hung, L., Gernot, P., Ronglin, W., Molly, D., et al. (2002). A draft sequence of the rice genome (oryza sativa L. ssp. japonica). Science 296, 1937-1942. doi: 10.1126/science.1068275

He, G. H., Xu, J. Y., Wang, Y. X., Liu, J. M., Li, P. S., Chen, M., et al. (2016). Drought-responsive WRKY transcription factor genes TaWRKY1 and TaWRKY33 from wheat confer drought and/or heat resistance in Arabidopsis. BMC Plant Biol. 16:116. doi: 10.1186/s12870-016-0806-4

He, Y., Mao, S., Gao, Y., Zhu, L., Wu, D., Cui, Y., et al. (2016). Genome-wide identification and expression analysis of WRKY transcription factors under multiple stresses in Brassica napus. PLoS ONE 11:e015755. doi: 10.1371/journal. pone. 0157558

Hinderhofer, K., and Zentgraf, U. (2001). Identification of a transcription factor specifically expressed at the onset of leaf senescence. Planta 213, 469-473. doi: $10.1007 / \mathrm{s} 004250000512$

Ishiguro, S., and Nakamura, K. (1994). Characterization of a cDNA encoding a novel DNA-binding protein, SPF1, that recognizes SP8 sequences in the 5' upstream regions of genes coding for sporamin and $\beta$-amylase from sweet potato. Mol. Gen. Genet. 244, 563-571. doi: 10.1007/BF00282746

Jin, C., Huang, X. S., Li, K. Q., Yin, H., Li, L. T., Yao, Z. H., et al. (2016). Overexpression of a bHLH1 transcription factor of Pyrus ussuriensis confers enhanced cold tolerance and increases expression of stress-responsive genes. Front. Plant Sci. 7:441. doi: 10.3389/fpls.2016.00441

Jin, X., Xue, Y., Wang, R., Xu, R., Bian, L., Zhu, B., et al. (2013). Transcription factor OsAP21 gene increases salt/drought tolerance in transgenic Arabidopsis thaliana. Mol. Biol. Rep. 40, 1743-1752. doi: 10.1007/s11033-012-2 228-1

Johnson, C. S., Kolevski, B., and Smyth, D. R. (2002). Transparent TESTA GLABRA2, a trichome and seed coat development gene of Arabidopsis, encodes a WRKY transcription factor. Plant Cell 14, 1359-1375. doi: 10.1105/tpc.001404

Lata, C., Mishra, A. K., Muthamilarasan, M., Bonthala, V. S., Khan, Y., and Prasad, M. (2014). Genome-wide investigation and expression profiling of AP2/ERF transcription factor superfamily in foxtail millet (Setaria italica L.). PLoS ONE 9:e113092. doi: 10.1371/journal.pone.0113092

Lavin, M., Herendeen, P. S., and Wojciechowski, M. F. (2005). Evolutionary rates analysis of Leguminosae implicates a rapid diversification of lineages during the tertiary. Syst. Biol. 54, 575-594. doi: 10.1080/10635150590947131

Lescot, M., Déhais, P., Thijs, G., Marchal, K., Moreau, Y., Van de Peer, Y., et al. (2002). PlantCARE, a database of plant cis-acting regulatory elements and a portal to tools for in silico analysis of promoter sequences. Nucleic Acids Res. 30, 325-327. doi: 10.1093/nar/30.1.325

Li, M. J., Qiao, Y., Li, Y. Q., Shi, Z. L., Zhang, N., Bi, C. L., et al. (2016). A R2R3-MYB transcription factor gene in common wheat (namely TaMYBsm1) involved in enhancement of drought tolerance in transgenic Arabidopsis. J. Plant Res. 129, 1097-1107. doi: 10.1007/s10265-016-0857-5

Li, S., Fu, Q., Chen, L., Huang, W., and Yu, D. (2011). Arabidopsis thaliana WRKY25, WRKY26, and WRKY33 coordinate induction of plant thermotolerance. Planta 233, 1237-1252. doi: 10.1007/s00425-011-1375-2
Li, S., Fu, Q., Huang, W., and Yu, D. (2009). Functional analysis of an Arabidopsis transcription factor WRKY25 in heat stress. Plant Cell Rep. 28, 683-693. doi: 10.1007/s00299-008-0666-y

Li, W., Wang, H., and Yu, D. (2016). Arabidopsis WRKY transcription factors WRKY12 and WRKY13 oppositely regulate flowering under short-day conditions. Mol. Plant 9, 1492-1503. doi: 10.1016/j.molp.2016.08.003

Liu, X. F., Song, Y. Z., Xing, F. Y., Wang, N., Wen, F. J., and Zhu, C. X. (2016). GhWRKY25, a group I WRKY gene from cotton, confers differential tolerance to abiotic and biotic stresses in transgenic Nicotiana benthamiana. Protoplasma 253, 1265-1281. doi: 10.1007/s00709-015-0885-3

Lozano, R., Hamblin, M. T., Prochnik, S., and Jannink, J. L. (2015). Identification and distribution of the NBS-LRR gene family in the cassava genome. BMC Genomics 16:360. doi: 10.1186/s12864-015-1554-9

Luo, M., Dennis, E. S., Berger, F., Peacock, W. J., and Chaudhury, A. (2005). MINISEED3 (MINI3), a WRKY family gene, and HAIKU2 (IKU2), a leucinerich repeat (LRR) kinase gene, are regulators of seed size in Arabidopsis. Proc. Natl Acad. Sci. U.S.A. 102, 17531-17536. doi: 10.1073/pnas.0508418102

Luo, X., Bai, X., Sun, X. L., Zhu, D., Liu, B. H., Ji, W., et al. (2013). Expression of wild soybean WRKY20 in Arabidopsis enhances drought tolerance and regulates ABA signalling. J. Exp. Bot. 64, 2155-2169. doi: 10.1093/jxb/ert073

Mao, H., Wang, H., Liu, S., Li, Z., Yang, X., Yan, J., et al. (2015). A transposable element in a NAC gene is associated with drought tolerance in maize seedlings. Nat. Commun. 6:8326. doi: 10.1038/ncomms9326

Mao, X., Chen, S., Li, A., Zhai, C., and Jing, R. (2014). Novel NAC transcription factor TaNAC67 confers enhanced multi-abiotic stress tolerances in Arabidopsis. PLoS ONE 9:e84359. doi: 10.1371/journal.pone.0084359

Mao, X., Zhang, H., Qian, X., Li, A., Zhao, G., and Jing, R. (2012). TaNAC2, a NACtype wheat transcription factor conferring enhanced multiple abiotic stress tolerances in Arabidopsis. J. Exp. Bot. 63, 2933-2946. doi: 10.1093/jxb/err462

Marè, C., Mazzucotelli, E., Crosatti, C., Francia, E., Stanca, A. M., and Cattivelli, L. (2004). Hv-WRKY38: a new transcription factor involved in cold- and droughtresponse in barley. Plant Mol. Biol. 55, 399-416. doi: 10.1007/s11103-0040906-7

Muthamilarasan, M., Khandelwal, R., Yadav, C. B., Bonthala, V. S., Khan, Y., and Prasad, M. (2014). Identification and molecular characterization of myb transcription factor superfamily in c4 model plant foxtail millet (Setaria italica L.). PLoS ONE 9:e109920. doi: 10.1371/journal.pone.0109920

Piao, S. L., Ciais, P., Huang, Y., Shen, Z. H., Peng, S. H., Li, J. S., et al. (2010). The impacts of climate change on water resources and agriculture in China. Nature 467, 43-51. doi: 10.1038/nature09364

Popescu, S. C., Popescu, G. V., Bachan, S., Zhang, Z., Gerstein, M., Snyder, M., et al. (2009). MAPK target networks in Arabidopsis thaliana revealed using functional protein microarrays. Genes Dev. 23, 80-92. doi: 10.1101/gad. 1740009

Qiao, L., Zhang, X., Han, X., Zhang, L., Li, X., Zhan, H., et al. (2015). A genomewide analysis of the auxin/indole-3-acetic acid gene family in hexaploid bread wheat (Triticum aestivum L.). Front. Plant Sci. 6:770. doi: 10.3389/fpls.2015. 00770

Qin, Y., Tian, Y., and Liu, X. (2015). A wheat salinity-induced WRKY transcription factor TaWRKY93 confers multiple abiotic stress tolerance in Arabidopsis thaliana. Biochem. Biophys. Res. Commun. 464, 428-433. doi: 10.1016/j.bbrc. 2015.06.128

Qiu, Y., and Yu, D. (2009). Over-expression of the stress-induced OsWRKY45 enhances disease resistance and drought tolerance in Arabidopsis. Environ. Exp. Bot. 65, 35-47. doi: 10.1016/j.envexpbot.2008.07.002

Ren, X. Z., Chen, Z. Z., Liu, Y., Zhang, H. R., Zhang, M., Liu, Q., et al. (2010). ABO3, a WRKY transcription factor, mediates plant responses to abscisic acid and drought tolerance in Arabidopsis. Plant J. 63, 417-429. doi: 10.1111/j.1365313X.2010.04248.x

Robatzek, S., and Somssich, I. E. (2001). A new member of the Arabidopsis WRKY transcription factor family, AtWRKY6, is associated with both senescence- and defence-related processes. Plant J. 28, 123-133. doi: 10.1046/j.1365-313X.2001. 01131.x

Ross, C. A., Liu, Y., and Shen, Q. J. (2007). The WRKY gene family in rice (Oryza sativa). J. Integr. Plant Biol. 49, 827-842. doi: 10.1111/j.1744-7909.2007.00504.x

Rushton, P. J., Somssich, I. E., Ringler, P., and Shen, Q. J. (2010). WRKY transcription factors. Trends Plant Sci. 15, 247-258. doi: 10.1016/j.tplants.2010. 02.006 
Schmutz, J., Cannon, S. B., Schlueter, J., Ma, J., Mitros, T., Nelson, W., et al. (2010). Genome sequence of the palaeopolyploid soybean. Nature 463, 178-183. doi: $10.1038 /$ nature08670

Schmutz, J., McClean, P. E., Mamidi, S., Wu, G. A., Cannon, S. B., Grimwood, J., et al. (2014). A reference genome for common bean and genome-wide analysis of dual domestications. Nat. Genet. 46, 707-713. doi: 10.1038/ng.3008

Song, H., Wang, P., Hou, L., Zhao, S., Zhao, C., Xia, H., et al. (2016a). Global analysis of WRKY genes and their response to dehydration and salt stress in soybean. Front. Plant Sci. 7:9. doi: 10.3389/fpls.2016.00009

Song, H., Wang, P., Lin, J. Y., Zhao, C., Bi, Y., and Wang, X. (2016b). Genome-wide identification and characterization of WRKY gene family in peanut. Front. Plant Sci. 7:534. doi: 10.3389/fpls.2016.00534

Song, H., Wang, P., Nan, Z., and Wang, X. (2014). The WRKY transcription factor genes in Lotus japonicus. Int. J. Genomics 3:420128. doi: 10.1155/2014/420128

Song, L., Huang, S. C., Wise, A., Castanon, R., Nery, J. R., Chen, H., et al. (2016). A transcription factor hierarchy defines an environmental stress response network. Science 354:aag1550. doi: 10.1126/science.aag1550

Song, Y., Chen, L., Zhang, L., and Yu, D. (2010). Overexpression of OsWRKY72 gene interferes in the abscisic acid signal and auxin transport pathway of Arabidopsis. J. Bio. Sci. 35, 459-471. doi: 10.1007/s12038-010-0051-1

Sun, C., Palmqvist, S., Olsson, H., Borén, M., Ahlandsberg, S., and Jansson, C. (2003). A novel WRKY transcription factor, SUSIBA2, participates in sugar signaling in barley by binding to the sugar-responsive elements of the isol promoter. Plant Cell 15, 2076-2092. doi: 10.1105/tpc.014597

Tao, Z., Liu, H., Qiu, D., Zhou, Y., Li, X., Xu, C., et al. (2009). A pair of allelic WRKY genes play opposite roles in rice-bacteria interactions. Plant Physiol. 151, 936-948. doi: 10.1104/pp.109.145623

The Arabidopsis Genome Initiative (2000). Analysis of the genome sequence of the flowering plant Arabidopsis thaliana. Nature 408, 796-815. doi: 10.1038/ 35048692

Ülker, B., and Somssich, I. E. (2004). WRKY transcription factors: from DNA binding towards biological function. Curr. Opin. Plant Biol. 7, 491-498. doi: 10.1016/j.pbi.2004.07.012

Vlasova, A., Capella-Gutiérrez, S., Rendón-Anaya, M., Hernández-Oñate, M., Minoche, A. E., Erb, I., et al. (2016). Genome and transcriptome analysis of the Mesoamerican common bean and the role of gene duplications in establishing tissue and temporal specialization of genes. Genome Biol. 17:32. doi: 10.1186/ s13059-016-0883-6

Wang, F., Chen, H. W., Li, Q. T., Wei, W., Li, W., Zhang, W. K., et al. (2015). GmWRKY27 interacts with GmMYB174 to reduce expression of GmNAC29 for stress tolerance in soybean plants. Plant J. 83, 224-236. doi: 10.1111/tpj.12879

Wang, X., Zeng, J., Li, Y., Rong, X., Sun, J., Sun, T., et al. (2015). Expression of TaWRKY44, a wheat WRKY gene, in transgenic tobacco confers multiple abiotic stress tolerances. Front. Plant Sci. 6:615. doi: 10.3389/fpls.2015.00615

Wang, Z., Li, P., Yang, Y., Chi, Y., Fan, B., and Chen, Z. (2016). Expression and functional analysis of a novel group of legume-specific WRKY and Exo70 protein variants from soybean. Sci. Rep. 6:32090. doi: 10.1038/srep32090

Wei, W., Hu, Y., Han, Y. T., Zhang, K., Zhao, F. L., and Feng, J. Y. (2016). The WRKY transcription factors in the diploid woodland strawberry Fragaria vesca: identification and expression analysis under biotic and abiotic stresses. Plant Physiol. Biochem. 105, 129-144. doi: 10.1016/j.plaphy.2016.04.014

Wu, J., Wang, L., Li, L., and Wang, S. (2014). De novo assembly of the common bean transcriptome using short reads for the discovery of drought-responsive genes. PLoS ONE 9:e109262. doi: 10.1371/journal.pone.0109262

Wu, J., Wang, L. F., and Wang, S. M. (2016). Comprehensive analysis and discovery of drought-related NAC transcription factors in common bean. BMC Plant Biol. 16:193. doi: 10.1186/s12870-016-0882-5

Wu, K. L., Guo, Z. J., Wang, H. H., and Li, J. (2005). The WRKY family of transcription factors in rice and Arabidopsis and their origins. DNA Res. 12, 9-26. doi: 10.1093/dnares/12.1.9
Wu, X., Shiroto, Y., Kishitani, S., Ito, Y., and Toriyama, K. (2009). Enhanced heat and drought tolerance in transgenic rice seedlings overexpressing OsWRKY11 under the control of HSP101 promoter. Plant Cell Rep. 28, 21-30. doi: 10.1007/ s00299-008-0614-x

Xie, Z., Zhang, Z. L., Zou, X. L., Huang, J., Ruas, P., Thompson, D., et al. (2005). Annotations and functional analyses of the rice WRKY gene superfamily reveal positive and negative regulators of abscisic acid signaling in aleurone cells. Plant Physiol. 137, 176-189. doi: 10.1104/pp.104.054312

Xiong, H., Li, J., Liu, P., Duan, J., Zhao, Y., Guo, X., et al. (2014). Overexpression of OsMYB48-1, a novel MYB-related transcription factor, enhances drought and salinity tolerance in rice. PLOS ONE 9:e92913. doi: 10.1371/journal.pone. 0092913

Xu, K., Chen, S., Li, T., Ma, X., Liang, X., Ding, X., et al. (2015). OsGRAS23, a rice GRAS transcription factor gene, is involved in drought stress response through regulating expression of stress-responsive genes. BMC Plant Biol. 15:141. doi: 10.1186/s12870-015-0532-3

Yang, Y., Chi, Y., Wang, Z., Zhou, Y., Fan, B., and Chen, Z. (2016). Functional analysis of structurally related soybean GmWRKY58 and GmWRKY76 in plant growth and development. J. Exp. Bot. 67, 4727-4742. doi: 10.1093/jxb/erw252

Yang, Z. (2007). PAML 4: phylogenetic analysis by maximum likelihood. Mol. Biol. Evol. 24, 1586-1591. doi: 10.1093/molbev/msm088

Yin, G., Xu, H., Xiao, S., Qin, Y., Li, Y., Yan, Y., et al. (2013). The large soybean (Glycine max) WRKY TF family expanded by segmental duplication events and subsequent divergent selection among subgroups. BMC Plant Biol. 13:148. doi: 10.1186/1471-2229-13-148

Young, N. D., Debellé, F., Oldroyd, G. E. D., Geurts, R., Cannon, S. B., Udvardi, M. K., et al. (2011). The Medicago genome provides insight into the evolution of rhizobial symbioses. Nature 480, 520-524. doi: 10.1038/nature10625

Yu, J., Hu, S., Wang, G. K., Li, S., Liu, B., Deng, Y., et al. (2002). A draft sequence of the rice genome (Oryza sativa L. ssp. indica). Science 296, 79-92. doi: 10.1126/ science. 1068037

Yue, H., Wang, M., Liu, S. Y., Du, X. H., Song, W. N., and Nie, X. J. (2016). Transcriptome-wide identification and expression profiles of the WRKY transcription factor family in Broomcorn millet (Panicum miliaceum L.). BMC Genomics 17:343. doi: 10.1186/s12864-016-2677-3

Zhang, Y., Yu, H., Yang, X., Li, Q., Ling, J., Wang, H., et al. (2016). CsWRKY46, a WRKY transcription factor from cucumber, confers cold resistance in transgenic-plant by regulating a set of cold-stress responsive genes in an ABAdependent manner. Plant Physiol. Biochem. 108, 478-487. doi: 10.1016/j.plaphy. 2016.08.013

Zhang, Z. L., Xie, Z., Zou, X., Casaretto, J., Ho, T. H., and Shen, Q. J. (2004). A rice WRKY gene encodes a transcriptional repressor of the gibberellin signaling pathway in aleurone cells. Plant Physiol. 134, 1500-1513. doi: 10.1104/pp.103. 034967

Zhou, Q. Y., Tian, A. G., Zou, H. F., Xie, Z. M., Lei, G., Huang, J., et al. (2008). Soybean WRKY-type transcription factor genes, GmWRKY13, GmWRKY21, and GmWRKY54, confer differential tolerance to abiotic stresses in transgenic Arabidopsis plants. Plant Biotechnol. J. 6, 486-503. doi: 10.1111/j.1467-7652. 2008.00336.x

Conflict of Interest Statement: The authors declare that the research was conducted in the absence of any commercial or financial relationships that could be construed as a potential conflict of interest.

Copyright (c) $2017 \mathrm{Wu}$, Chen, Wang and Wang. This is an open-access article distributed under the terms of the Creative Commons Attribution License (CC BY). The use, distribution or reproduction in other forums is permitted, provided the original author(s) or licensor are credited and that the original publication in this journal is cited, in accordance with accepted academic practice. No use, distribution or reproduction is permitted which does not comply with these terms. 